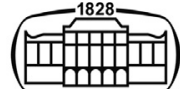

AKADÉMIAI KIADÓ

\title{
Biofilm-forming lactic acid bacteria of honey bee origin intended for potential probiotic use
}

\section{Acta Veterinaria Hungarica}

$68(2020) 4,345-353$

DOl:

10.1556/004.2020.00057

(c) 2021 Akadémiai Kiadó, Budapest

\section{ORIGINAL RESEARCH} PAPER

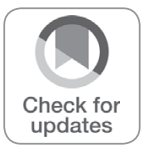

*Corresponding author.

E-mail: dagmar.mudronova@uvlf.sk
BOHUMIL BIELIK ${ }^{1}$, LADISLAV MOLNÁR ${ }^{1}$, VLADIMÍR VRABEC ${ }^{1}$, ROMANA ANDRÁŠIOVÁ ${ }^{2}$, IVANA CINGEL'OVÁ MARUŠČĆKOVÁ ${ }^{3}$, RADOMÍRA NEMCOVÁ ${ }^{3}$, JURAJ TOPORČÁK ${ }^{1}$ and DAGMAR MUDROÑOVÁ ${ }^{3 *}$

\footnotetext{
${ }^{1}$ Clinic of Birds, Exotic and Free Living Animals, University of Veterinary Medicine and Pharmacy, Košice, Slovak Republic

${ }^{2}$ Department of Epizootiology and Parasitology, University of Veterinary Medicine and Pharmacy, Košice, Slovak Republic

${ }^{3}$ Department of Microbiology and Immunology, University of Veterinary Medicine and Pharmacy, Komenského 73, 041 81, Košice, Slovak Republic
}

Received: July 23, 2020 • Accepted: October 14, 2020

Published online: January 25, 2021

\begin{abstract}
Scientists around the world are focusing their interest on the use of probiotics in honey bees as an alternative method of prophylaxis against causative agents of both American and European foulbrood. In our study we tested inhibitory activity against Paenibacillus larvae and the biofilm formation activity by various lactic acid bacteria isolated from honey bee guts or fresh pollen samples in the presence of different sugars added to the cultivation media. In addition, we tested the probiotic effect of a newly selected Apilactobacillus kunkeei V18 in an in situ experiment in bee colonies. We found antibacterial activity against $P$. larvae in four isolates. Biofilm formation activity of varying intensity was noted in six of the seven isolates in the presence of different sugars. The strongest biofilm formation $\left(\mathrm{OD}_{570} \geq 1\right)$ was noted in A. kunkeei V18 in the presence of fructose; moreover, this isolate strongly inhibited the growth of $P$. larvae under laboratory conditions. Inhibition of $P$. larvae and Melissococcus plutonius by A. kunkeei V18 in situ was confirmed in a pilot study.
\end{abstract}

\section{KEYWORDS}

probiotic, honey bee, biofilm, fructose, Apilactobacillus kunkeei

\section{INTRODUCTION}

Lactic acid bacteria (LAB) are a group of Gram-positive, non-spore-forming cocci or rods which produce lactic acid as the major product of carbohydrate fermentation. These bacteria are common commensals of the gut and other body surfaces in humans, animals and insects (Mathialagan et al., 2018). Moreover, they have the ability to produce different types of exopolysaccharides which are classified, according to their composition, as homopolysaccharides and heteropolysaccharides (Badel et al., 2011). Bacterial exopolysaccharides offer a large set of physicochemical and biological properties. These include adhesives hiding the bacterial surface, adhesiveness for interactions with other bacterial surfaces, protection against environmental or antibiotic effects, and structural stabilisation in biofilm (Sutherland, 1972). The presence of different extracellular saccharides in a bacterial environment can cause changes in the physicochemical properties of a constant surface, which in turn leads to indirect changes in the formation of biofilms (Khangholi and Jamalli, 2016). The term biofilm is used to describe a structured community of bacterial cells enclosed in a self-produced 
polymeric matrix that is adherent to an inert or living surface (Costerton et al., 1999). Biofilm is involved in bacterial communication and defence mechanisms. In addition, it can protect microorganisms against several stress conditions as well as antimicrobial agents (Simoes et al., 2009). Biofilmlike communities of the gastrointestinal tract containing beneficial lactobacilli may have a protective role (Kubota et al., 2008). Survivability and colonisation in the digestive tract are regarded as critical factors for the optimal healthpromoting physiological function of probiotics (Kaushik et al., 2009).

Probiotic bacteria are defined as live microorganisms which, when administered to a host in adequate amounts, confer them health benefits' (FAO/WHO, 2002). These organisms must tolerate low $\mathrm{pH}$ and other conditions to survive in the gut (Ramasamy et al., 2010). The characteristics that microorganisms must posses to be called effective probiotics may include their capacity to increase the number of beneficial microorganisms in the gastrointestinal tract and, at the same time, to decrease the impact of pathogenic microorganisms present without causing alterations that will trigger pathology. After proving that potential probiotic strains are safe, and that they possess antimicrobial activity, assays in cellular and animal models are performed. Finally, when all requested requirements are fulfilled, it is possible to start production of the probiotic product (Lee and Salminen, 2008).

The use of probiotics in honey bees has become more common in recent years. Preventive application of antibiotics to livestock has been banned in the EU since 2006 (Regulation 1831/2003/EC; European Commission, 2003). Unlike some countries in North and South America or Asia, the therapeutic use of antimicrobials in honey bees is forbidden in many EU countries by relevant laws (OIE, 2013; e.g. Law No. 39/2007 Coll. of the National Council of the Slovak Republic on Veterinary Care). Probiotics appear to be an effective method to combat infectious diseases such as European and American foulbrood. The inhibitory effect of probiotics against Paenibacillus larvae, the causative agent of American foulbrood, has been described in many studies (Alippi and Reynaldi, 2006; Forsgren et al., 2010; Mudroňová et al., 2011; Al-Ghamdi et al., 2018).

However, the development of probiotic products should not be limited to the inhibition of pathogens. Microbial safety of the honey bee products, treatment duration, seasonality of use, cost-effectiveness, and ease of use for beekeepers are the main factors which must also be taken into account during the development of probiotic preparations (Alberoni et al., 2016).

Based on this information we decided to test the biofilmforming activity of LAB isolated from the gut of honey bees and fresh pollen samples in the presence of different sugars in media, to test their antibacterial activity against $P$. larvae in vitro and then to prepare a probiotic preparation with the potential probiotic strain showing the best properties and test it in a pilot experiment on $P$. larvae-positive honey bee colonies.

\section{MATERIALS AND METHODS}

\section{Microorganisms and growth conditions}

The strains of LAB used in the present study were isolated from the digestive tracts of healthy adult honey bees at the Department of Microbiology and Immunology of the University of Veterinary Medicine and Pharmacy in Košice, Slovakia. Bee guts $(0.5 \mathrm{~g})$ were crushed in sterile saline (4.5 $\mathrm{mL}$ ) and inoculated onto de Man-Rogosa-Sharpe agar (MRS; Merck, Germany) and grown at $37{ }^{\circ} \mathrm{C}$ anaerobically (Gas Pak Plus, BBL Microbiology Systems, USA) for $48 \mathrm{~h}$. Other strains were isolated from fresh pollen samples $(0.5 \mathrm{~g}$ of sample dissolved in $4.5 \mathrm{~mL}$ of saline). Overall, four strains were isolated from the gut of adult bees (isolates V12, V18, $\mathrm{V} 19, \mathrm{Z1}$ ), and two strains from pollen samples (P3, P4). All strains were identified biochemically using $\mathrm{API}^{\circledR} 50 \mathrm{CH}$ test (BioMérieux, France). The tests were performed according to the manufacturer's instructions. The autochthonous honey bee probiotic strain Lactobacillus brevis B50 Biocenol (CCM 8618) described in our previous paper (Mudroňová et al., 2011) was also included. This strain, when applied to hives, showed strong inhibitory activity against $P$. larvae, improved the condition and immunity of bee colonies and also stimulated the production of antimicrobial peptides in the intestines of bees (Kuzyšinová et al., 2012; Marušč́ková et al., 2020).

The identification of Apilactobacillus kunkeei V18 (formerly designated as Lactobacillus kunkeei) was confirmed using MALDI-TOF MS. A single colony from MRS agar was used for the analysis, and the preparation was performed according to Ferreira et al. (2011). Data (raw spectra) received from Microflex LT instrument (Bruker Daltonics $\mathrm{GmbH}$, Germany) using FlexControl software (version 3.0) was imported into Biotyper software version 3.0 (Bruker Daltonics GmbH, Germany, database version 3.3.1.0) and analysed by standard pattern matching with default settings without any user intervention.

\section{In vitro inhibitory activity against Paenibacillus larvae}

Inhibitory activity of $\mathrm{LAB}$ bee isolates was tested using modified disc assay. Sterile paper discs (6 $\mathrm{mm}$ in diameter; BBL Microbiology Systems, Becton Dickinson, USA) were placed onto the surface of MRS agar $(20 \mathrm{~mL} /$ plate $)$ and 10 $\mu \mathrm{L}$ of the appropriate isolate was pipetted onto the discs. The plates were incubated as described above for $48 \mathrm{~h}$. Then the discs were removed from the surface of MRS agar and the plates were exposed to chloroform vapour for $30 \mathrm{~min}$, then the vapour was exhausted over a 2 -h period. The plates were overlaid with $3 \mathrm{~mL}$ of $0.7 \%$ MYPGP agar (yeast extract $15 \mathrm{~g}$, $\mathrm{K}_{2} \mathrm{HPO}_{4} 3 \mathrm{~g}$, sodium pyruvate $1 \mathrm{~g}$, $\mathrm{D}(+)$ glucose $2 \mathrm{~g}$, agar for bacteriology $7 \mathrm{~g} \mathrm{~L}^{-1}, \mathrm{pH} 7.2$ ), and inoculated with $300 \mu \mathrm{L}$ of overnight culture of $P$. larvae CCM 4488 and incubated aerobically at $37^{\circ} \mathrm{C}$ for $24 \mathrm{~h}$. After incubation, the diameters of the inhibition zones were measured. For each strain an arithmetic mean of zone diameters from three measurements was calculated. The tested isolates were divided into 
non-inhibiting to weakly inhibiting $(<10 \mathrm{~mm})$, moderately inhibiting $(10 \mathrm{~mm} \leq$ zone $\leq 16 \mathrm{~mm})$ and strongly inhibiting (>16 mm).

\section{In vitro biofilm assay}

Modified MRS broth (peptone $10 \mathrm{~g}$, beef extract $8 \mathrm{~g}$, yeast extract $5 \mathrm{~g}$, sugar $20 \mathrm{~g}$, sodium acetate trihydrate $3 \mathrm{~g}$, $\mathrm{K}_{2} \mathrm{HPO}_{4} 2 \mathrm{~g}$, triammonium citrate $2 \mathrm{~g}, \mathrm{MgSO}_{4} 0.2 \mathrm{~g}, \mathrm{MnSO}_{4}$ $0.05 \mathrm{~g} \mathrm{~L}^{-1}, \mathrm{pH}$ 6.2) with the addition of different sugars (Dglucose, sucrose, lactose, D-fructose, maltodextrin, and maltose; Sigma-Aldrich, USA) was used for biofilm assay. A modified version of the previously described method (O’Toole et al., 1999) was used to test biofilm formation. Fifty $\mu \mathrm{L}$ of bacterial suspension (McFarland standard 1) was transferred to $5 \mathrm{~mL}$ of appropriate sugar-containing broth, and subsequently $200 \mu \mathrm{L}$ of this culture was pipetted into the microtitre plates (Greiner ELISA 8 Well Strips, $350 \mu \mathrm{L}$, Flat Bottom, Medium Binding; Cruinn Diagnostics Ltd., Ireland) and incubated at $37{ }^{\circ} \mathrm{C}$ for $24 \mathrm{~h}$. After incubation the supernatant was gently removed by aspiration, and the individual wells were washed with distilled water 5 times. After washing and drying at room temperature for $30 \mathrm{~min}$, the biofilm was stained with $0.1 \%$ crystal violet in an isopropanol-methanol-PBS solution $(1: 1: 18 \mathrm{~V} / \mathrm{V})(200 \mu \mathrm{L}$ per well, $30 \mathrm{~min}$, room temperature). Then the crystal violet was aspirated again, and the wells were washed thoroughly with distilled water three times. After drying for $30 \mathrm{~min}$ at room temperature, the dye bound to the adherent biofilm was extracted using $200 \mu \mathrm{L}$ of $30 \%$ acetic acid per well. An aliquot of $150 \mu \mathrm{L}$ from each well was taken and placed in a new microplate for OD determination at $570 \mathrm{~nm}$ using the Synergy $^{\mathrm{TM}} 4$ Multi-Mode Microplate Reader (BioTek, USA). Each strain was tested in three independent experiments with eight biological replicates. Additionally, a sterile culture medium was always included as negative control to ensure that the influence of the biofilm formation was not attributed to a nonspecific binding effect to crystal violet. Biofilm formation was categorised as highly positive $\left(\mathrm{OD}_{570} \geq 1\right)$, low-grade positive $\left(0.1 \leq \mathrm{OD}_{570}<1\right)$, or negative $\left(\mathrm{OD}_{570}<\right.$ 0.1 ). The results are expressed as means \pm standard deviations (SD).

\section{Determination of the growth dynamics of $A$. kunkeei V18 in the presence of different sugars}

The growth dynamics of $A$. kunkeei V18 were evaluated in the presence of sucrose, lactose, and fructose added to the modified MRS broth (as described above). A volume of $50 \mu \mathrm{L}$ of the bacterial suspension (McFarland standard 1) was transferred to $5 \mathrm{~mL}$ of appropriate broth. The culture $(200 \mu \mathrm{L})$ was pipetted into the microtitre plate (Greiner ELISA 8 Well Strips, $350 \mu \mathrm{L}$, Flat Bottom, Medium Binding; Cruinn Diagnostics Ltd., Dublin, Ireland) and incubated at $37{ }^{\circ} \mathrm{C}$ for $24 \mathrm{~h}$. Optical density (OD) at $570 \mathrm{~nm}$ using Synergy $^{\mathrm{TM}} 4$ Multi-Mode Microplate Reader (BioTek, USA) was measured every $4 \mathrm{~h}$. The isolate was tested in three independent experiments with eight biological replicates. The results are expressed as means \pm SD.

\section{Preparation of diet supplements for a pilot in vivo study}

Pure pollen suspension was prepared from $50 \mathrm{~g}$ of dried multifloral bee pollen from a pesticide-free area and $450 \mathrm{~mL}$ of autoclaved tap water. For the preparation of probiotic supplement, A. kunkeei V18 was cultivated in MRS broth (Merck, Germany) at $37^{\circ} \mathrm{C}$ overnight. The overnight culture of lactobacilli was centrifuged at $700 \mathrm{~g}$ for $15 \mathrm{~min}$ and the sediment was washed twice in sterile saline solution. The prepared pellet was resuspended in pollen suspension containing also $1 \%$ of $\mathrm{D}$-fructose to stimulate growth and biofilm formation and the final concentration of lactobacilli was $10^{8}-10^{9} \mathrm{CFU} / \mathrm{mL}$.

\section{Fast testing for the presence of the causative agents of American and European foulbrood}

All colonies were tested at each collection for the presence of P. larvae with the AFB test kit (Vita, UK) and also for the appearance of Melisococcus plutonius, the causative agent of European foulbrood (EFB) with the EFB test kit (Vita, UK). The tests were performed on 5 samples from the affected combs according to the manufacturer's instructions. The presence of $P$. larvae was confirmed by microbiological cultivation on MYPGP agar as described below.

\section{Experimental design and sampling}

Twelve colonies from one apiary in eastern Slovakia were selected for a pilot in vivo study, where the presence of $P$. larvae was detected in adult bees, but without clinical signs of American foulbrood (AFB). The experiment was performed during the summer season (June-July). These hives were divided into three groups. The probiotic group was fed A. kunkeei V18 in pollen suspension $(n=4)$. Pure pollen suspension was applied to the pollen group $(n=4)$ and the control group did not receive any supplement $(n=$ 4). Supplements were applied to the colonies directly into the empty honeycombs, once a week for three subsequent weeks. The application dose for one colony was $500 \mathrm{~mL}$ of pollen suspension with or without lactobacilli. Samples were collected before the first application of supplements (0th sampling) and then every week for 3 weeks (1st-3rd sampling). Samples consisted of approximately 20 young adult bees from each colony. The digestive tracts of bees $(0.5 \mathrm{~g})$ were extracted for microbiological cultivation.

\section{Microbiological analysis}

Homogenised samples of digestive tracts were diluted with saline solution and plated onto MRS agar (Merck, Germany) to determine counts of LAB, Endo agar (Himedia, India) to count enterobacteria (ENT), blood agar composed of Columbia agar (Oxoid, UK) with 5\% defibrinated sheep blood to count total aerobes, and MYPGP agar to detect the presence of $P$. larvae. After inoculation, the MRS plates were incubated anaerobically (GasPak Becton Industries, UK) and also aerobically at $37^{\circ} \mathrm{C}$ for $48 \mathrm{~h}$ for counting A. kunkeei. 
Endoagar and blood agar were incubated aerobically at $37^{\circ} \mathrm{C}$ for $24 \mathrm{~h}$ and MYPGP plates aerobically at $37^{\circ} \mathrm{C}$ for 5 days. The bacterial counts were expressed in $\log _{10}$ of $\mathrm{CFU} / \mathrm{g}$ of intestinal content \pm SD.

\section{Statistical analysis}

The data were analysed with GraphPadPrism version 3.00 (GraphPad Software, USA). For the evaluation of biofilm assay, one-way analysis of variance (ANOVA) was used, followed by post-hoc Tukey's multiple comparison test. For other analyses two-way ANOVA was used, with subsequent post-hoc Tukey's test to analyse statistical differences in time and between groups.

\section{RESULTS}

\section{Identification of microorganisms}

The isolates obtained from bee guts were identified by the $\mathrm{API}^{\circledR} 50 \mathrm{CH}$ test as Streptococcus salivarius subsp. thermophilus V12, Apilactobacillus kunkeei V18, Levilactobacillus brevis V19, Lactobacillus delbrueckii subsp. delbrueckii Z1, and the isolates from pollen as Lactobacillus brevis P3 and Leuconostoc mesenteroides subsp. mesenteroides P4. The identification of A. kunkeei V18 was confirmed by MALDITOF MS with a score value of 2.136 , whereby scores $\geq 2.0$ are considered high-confidence (secure) species identification.

\section{Inhibition of Paenibacillus larvae}

The inhibitory activity of honey bee LAB against $P$. larvae CCM 4488 differed significantly among isolates. Three isolates showed no antimicrobial activity against the pathogen, while three others, and the probiotic strain L. brevis B50, were found to exert a strong inhibitory effect on P. larvae (Table 1).

\section{In vitro biofilm assay}

The formation of biofilm differed among the tested isolates of LAB and in the presence of different sugars. S. salivarius subsp. thermophilus V12 showed medium-degree production of biofilm $\left(\mathrm{OD}_{570}=0.11\right)$ after the addition of maltose to the medium, but not after the addition of other sugars (Fig. 1a). High ability to form biofilm $\left(\mathrm{OD}_{570} \geq 1\right)$ was observed in A. kunkeei V18 in MRS broth supplemented with fructose, which was significantly higher in comparison with all other sugars tested $(P>0.001)$. Medium-degree biofilm-forming activity was found after the addition of lactose, glucose and maltodextrin to the broth, and no biofilm-forming activity was noted in the presence of sucrose and maltose (Fig. 1b). The addition of glucose and fructose to the media caused a moderate level of biofilm formation in L. brevis V19, while other sugars did not have this ability (Fig. 1c). Lactobacillus delbrueckii subsp. delbrueckii Z1 formed a biofilm only in the broth supplemented with sucrose (Fig. 1d). Lactobacillus brevis $\mathrm{P} 3$ and $L$. brevis $\mathrm{B} 50$ Biocenol $^{\mathrm{TM}}$ CCM 8618 showed a middle-degree production of biofilm in the presence of maltose (Fig. 1e and f). Leuconostoc mesenteroides subsp.
Table 1. Antibacterial activity of honey bee LAB isolates against Paenibacillus larvae CCM4488 expressed as an average of inhibition zone diameters in $\mathrm{cm} \pm$ standard deviation (SD) $(n=3)$ Diameter of inhibition Isolate zones $(\mathrm{cm} \pm \mathrm{SD})$

\begin{tabular}{lc}
\hline $\begin{array}{l}\text { Streptococcus salivarius subsp. } \\
\text { thermophilus } \mathrm{V} 12\end{array}$ & 0 \\
Apilactobacillus kunkeei $\mathrm{V} 18$ & $4.2 \pm 0.12$ \\
Levilactobacillus brevis $\mathrm{V} 19$ & 0 \\
Lactobacillus delbrueckii subsp. & 0 \\
$\quad$ delbrueckii $\mathrm{Z} 1$ & $4.3 \pm 0.17$ \\
Lactobacillus brevis $\mathrm{P} 3$ & $3.3 \pm 0.17$ \\
Leuconostoc mesenteroides subsp. & \\
$\quad$ mesenteroides $\mathrm{P} 4$ & $4.6 \pm 0.14$ \\
Lactobacillus brevis B50 &
\end{tabular}

mesenteroides $\mathrm{P} 4$ was not able to form biofilm in the presence of any of the sugars tested (Fig. 1g).

\section{The growth dynamics of $A$. kunkeei V18 with fructose, sucrose and lactose}

The best growth of the isolate was noted in the presence of fructose with the highest growth speed between the 12th and 20th hour. The growth dynamics found in the presence of lactose were similar up to the 16th hour, then the isolate grew significantly slower than in the presence of fructose (at both 20 and $24 \mathrm{~h} ; P>0.001$ ). The weakest growth of A. kunkeei V18 was observed after the addition of sucrose to the medium (log phase between the 20th and the 24th h; Fig. 2).

\section{Microbiological screening in the pilot in vivo study}

The influence of administration of the probiotic preparation and pure pollen suspension on the intestinal microbiota of honey bees was evaluated on the basis of the counts of LAB incubated anaerobically and also aerobically (for the evaluation of A. kunkeei), the counts of ENT and the counts of total aerobic bacteria. In all three samplings we noted significantly lower counts of ENT and total aerobes in the probiotic group than in the pollen and the control groups (Fig. $3 \mathrm{a}$ and $\mathrm{b}$ ). Between the 0th and the 1st sampling, in the probiotic group we recorded a significant decrease of ENT and total aerobe counts (in both cases: $P<0.001$ ) and, on the other hand, an increase of LAB counts, especially aerobically cultured LAB counts, which correspond to the representation of A. kunkeei (Fig. 3c and d). The LAB to ENT ratio is a widely recognised indicator of intestinal microbial health. We observed a significantly higher ratio $(P<0.001)$ at all three post-application samplings in the probiotic group than in the control and the pollen groups (Fig. 3e). The most important finding was that $P$. larvae was not detectable on MYPGP agar from the 2nd sampling in the probiotic group.

\section{Fast testing for AFB and EFB}

Before the experiment (0th sampling), the presence of $P$. larvae was confirmed by the AFB test in all colonies. 
a

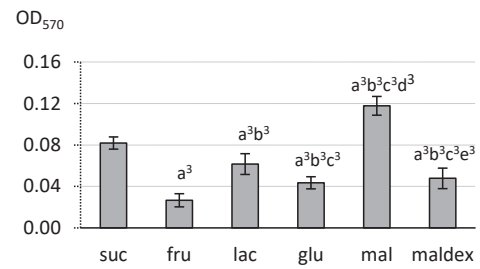

C

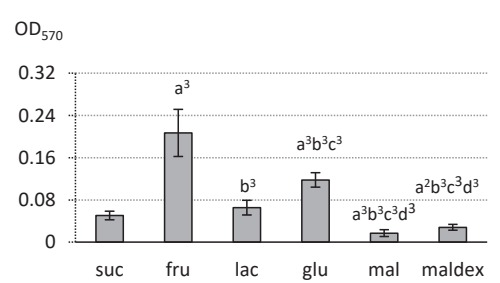

e

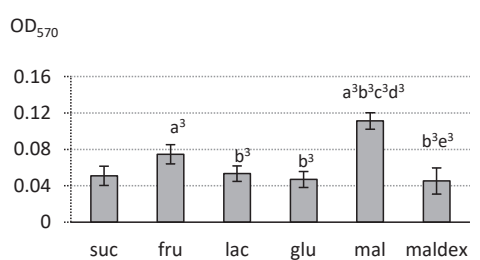

b

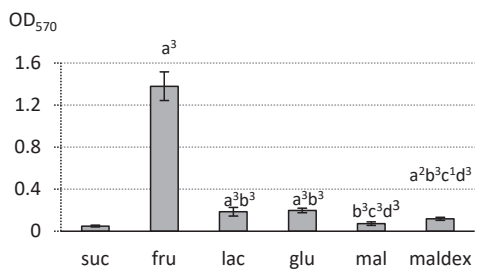

d

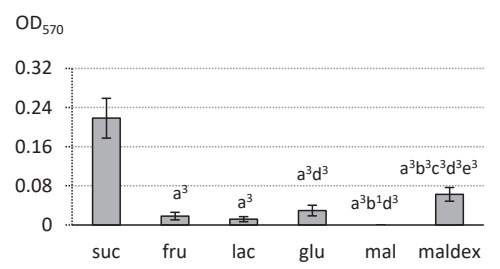

f

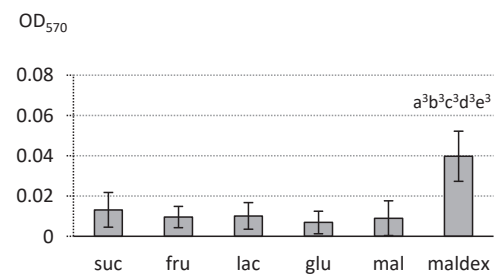

g

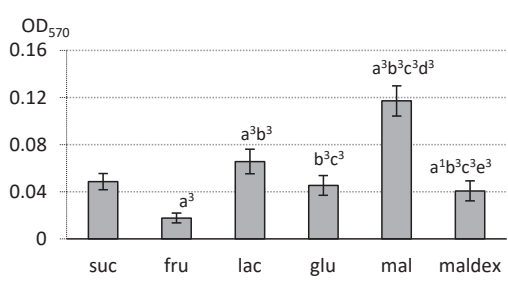

Fig. 1. Biofilm-forming activity $\left(\mathrm{OD}_{570 \mathrm{~nm}}\right)$ lactic acid bacteria isolated from of honey bee and pollen: (a) Streptococcus salivarius subsp. thermophilus V12, (b) Apilactobacillus kunkeei V18, (c) Levilactobacillus brevis V19, (d) Lactobacillus delbrueckii subsp. delbrueckii Z1, (e) Lactobacillus brevis P3, (f) Lactobacillus brevis B50, and (g) Leuconostoc mesenteroides subsp. mesenteroides P4. Statistical differences: significantly different from: $a$ - sucrose, $b$ - fructose, $c$ - lactose, $d$ - glucose, $e-$ maltose, $f$ - maltodextrin; ${ }^{1}-P>0.05,{ }^{2}-P>0.01,{ }^{3}-P>0.001$

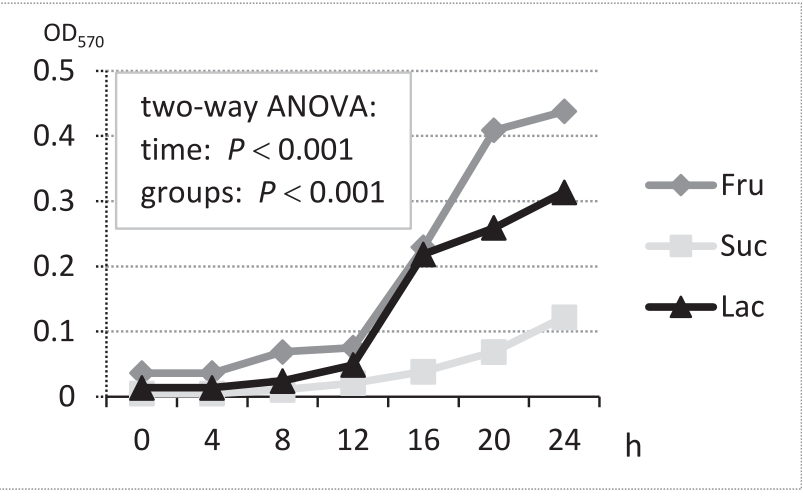

Fig. 2. Growth dynamics of Apilactobacillus kunkeei V18 in the presence of fructose, sucrose or lactose in the cultivation medium
Moreover, in one colony we detected M. plutonius and this colony was included in the probiotic group. Like the cultivation, the AFB test was repeatedly negative in all probiotic group hives on days 14 and 21 after the first application of the probiotic supplement. In the control and the pollen groups, the presence of the pathogen was detected until the end of the experiment. Interestingly, M. plutonius also disappeared from the digestive tract of bees after 3 weeks of administration of the probiotic preparation, and the EFB test was negative.

\section{DISCUSSION}

There is a growing interest in the use of probiotics as an alternative method for the prevention of infectious honey 
a

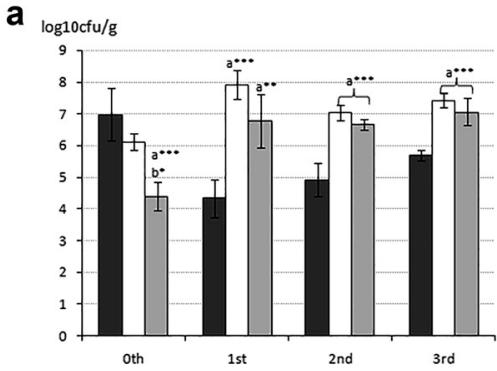

C

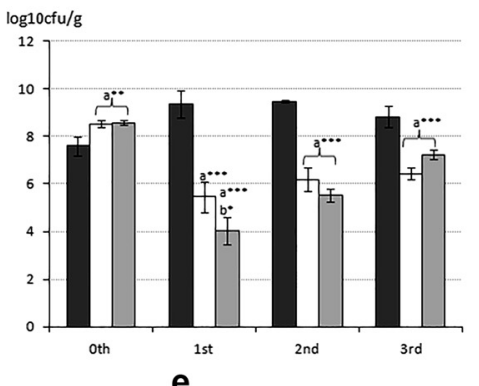

b

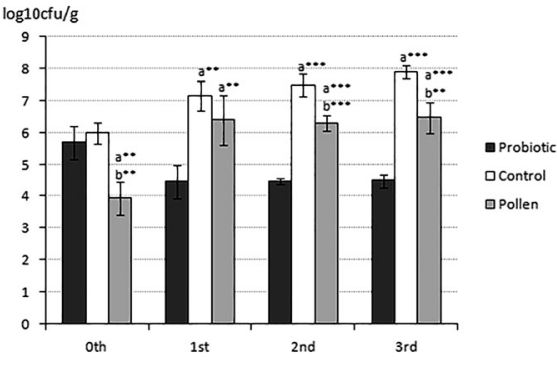

d

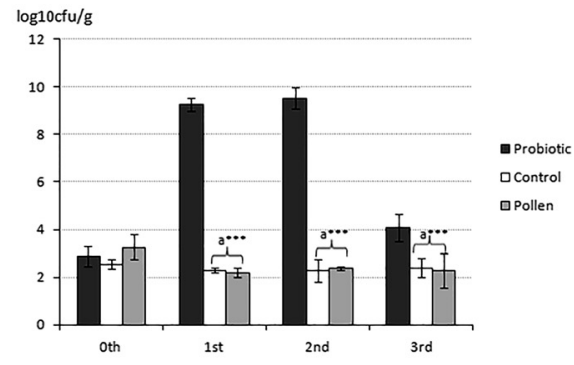

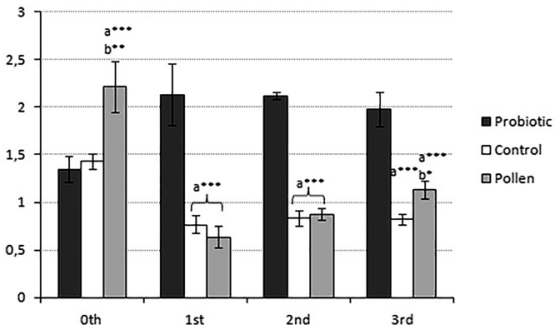

Fig. 3. Influence of the application of probiotic Apilactobacillus kunkeei V18 in pollen suspension and pure pollen suspension on the counts of: (a) total aerobes, (b) enterobacteria, (c) lactic acid bacteria (LAB), (d) aerobically growing LAB corresponding to A. kunkeei expressed as

$\log _{10} \mathrm{cfu} / \mathrm{g}$, and (e) ratio of LAB to ENT in the digestive tracts of honey bees. a - significantly different from the probiotic group, $\mathrm{b}-$ significantly different from the control group; ${ }^{*}-P<0.05,{ }^{* *}-P<0.01,{ }^{* * *}-P<0.001$

bee diseases, such as the American or European foulbrood (Daisley et al., 2020). Probiotics are able to influence the composition of the gut microbiome, and the immune response of honey bees is similar to that of mammals (Maruščáková et al., 2020). However, the efficacy of probiotics is closely related to their ability to inhibit the pathogens. Many authors have confirmed the antagonistic action of different bacterial species against $P$. larvae, the causative agent of American foulbrood (Alippi and Reynaldi, 2006; Forsgren et al., 2010; Mudroňová et al., 2011; Al-Ghamdi et al., 2018). The strong antibacterial activity was already confirmed in probiotic strain L. brevis B50 Biocenol (CCM 8618), which was used in this study as a reference strain. We found out that two tested isolates - A. kunkeei V18 and L. brevis $\mathrm{P} 3$ - possess a comparatively strong inhibitory activity similar to $L$. brevis B50, while Leu. mesenteroides subsp. mesenteroides $\mathrm{P} 4$ showed significantly weaker but still strong anti-P. larvae activity $(P<0.01-0.001)$. The other isolates $-S$. salivarius subsp. thermophilus V12, Lev. brevis V19 and L. delbrueckii subsp. delbrueckii Z1 - did not inhibit $P$. larvae.

Another critical requirement for a good probiotic strain is its ability to colonise and survive under the conditions of the host's digestive tract. Biofilm formation by probiotic bacteria is considered to be a beneficial property, since it can promote colonisation and prolong persistence on the gut mucosa of the host by avoiding colonisation by pathogenic bacteria (Terraf et al., 2012). The formation of biofilm by a probiotic strain favours the development of new technologies, e.g. encapsulation to maintain the viability of probiotics and allow them to arrive in intact form to their target site, or lyophilisation, which helps to prolong the storage of probiotics. A study performed by Olson et al. (2018) demonstrated a significant increase in biofilm formation by Lactobacillus reuteri on dextranomer microspheres by adding maltose and sucrose to the substrate. We noted biofilm activity with maltose added to the broth with three isolates: S. salivarius subsp. thermophilus V12, L. brevis B50 Biocenol $^{\mathrm{TM}}$ and $L$. brevis $\mathrm{P} 3$; on the other hand, $L$. delbrueckii subsp. delbrueckii $\mathrm{Z1}$ was not able to grow in the medium with added maltose, but in the MRS broth supplemented with sucrose evident biofilm formation activity was recorded $\left(\mathrm{OD}_{570}=0.21\right)$. Physicochemical conditions (temperature, $\mathrm{pH}$, sugar compounds) are effective facilitators of biofilm formation (Giovannacci et al., 2000). In a study performed by Yang et al. (2006), which focused on the effect of sugars and antimicrobial substances on oral microbial biofilm formation, the authors demonstrated that sucrose increased biofilm formation more significantly than glucose, fructose, 
galactose, and lactose. In our study we found strong biofilm formation $\left(\mathrm{OD}_{570}=1.3\right)$ by $A$. kunkeei V18 after the addition of fructose to the MRS broth. Such strong biofilmforming activity in the fructose-containing medium can be explained by the fact that $A$. kunkeei belongs to the group of fructophilic lactic acid bacteria (FLAB). The species is differentiated from other lactobacilli based on its poor growth in glucose-containing media, better growth in the presence of oxygen, and the production of high concentrations of acetate from the metabolism of fructose (Maeno et al., 2016). Our strain V18 grows very poorly under anaerobic conditions, and therefore we had to determine its counts after aerobic cultivation. In addition, we tested the growth of A. kunkeei V18 for $24 \mathrm{~h}$ with sucrose, fructose, and lactose added to the medium. We detected statistically significant differences in time and also between sugars $(P>0.001)$. The best growth of the isolate was recorded in the presence of fructose, while the worst in the medium supplemented with sucrose. The log phase of growth was between the 12th and 16th hours with fructose and lactose.

Although lactose-derived prebiotics have unique biological and functional values, and they are also confirmed as 'safe' for use in humans and animals (Nath et al., 2018), using lactose as a prebiotic factor is excluded in honey bees because of its toxicity to the bee colonies, which was confirmed in a study performed by Peng (1981), where significantly higher mortality $(P<0.05)$ occurred in bees fed with lactose than in control bees. The failure of bees to utilise lactose therefore suggests the absence of emulsion (Phillips, 1927). Although lactose can also be found in the pollen in small amounts, it does not cause mortality of honey bees in nature, because the toxic dose is diluted to safe levels by sucrose (Barker, 1977). In our experiment, we observed biofilm formation after adding lactose to the growth medium only in the case of A. kunkeei V18, but because of the toxicity of lactose to bees, we do not recommend its use in probiotic preparations intended for honey bees.

In the study performed by Leathers and Bischoff (2011) investigating the biofilm-forming activity of various Leuconostoc spp., the authors proved that not every strain of Leu. mesenteriodes is capable of producing biofilm. This feature can be explained by the study performed by Badel et al. (2011) which showed a crucial role of dextran, which is required for biofilm formation. In our research, the isolate of Leu. mesenteroides subsp. mesenteroides $\mathrm{P} 4$ did not show biofilm formation activity in the presence of any of the sugars tested.

Based on previous results, we decided to perform a pilot in vivo study with the strain A. kunkeei V18 showing the best probiotic properties and biofilm-forming activity in the presence of fructose. For these reasons, we applied the probiotic suspension in situ to the colonies where we recorded the occurrence of $P$. larvae, but without clinical signs. We even isolated M. plutonius in one hive. Due to the organisational structure of the bee colonies in modern beekeeping, the transmission of diseases is rapid, mainly through infected food or via nurse worker bees which feed the brood; therefore, reduction of the pathogenic microbiota in adult bees also leads to a reduction in brood infections. Patruica and Mot (2012), who fed honey bees with the probiotic strain Lactobacillus acidophilus LA-14 for three weeks, observed a significant reduction in total aerobe counts in the digestive tracts of bees, accompanied by a significant increase in lactobacilli as compared with the control. In our study we also detected a significant reduction of enterobacteria and total aerobe counts and a significant increase of LAB in the probiotic group in comparison with the control bees and with the group fed pure pollen suspension. The LAB to ENT ratio was also significantly higher in the probiotic group $(P<0.001)$ at all three post-application samplings. Daisley et al. (2020) studied the effect of $A$. kunkeei BR-1 administered to bees experimentally infected with $P$. larvae, and they confirmed the reduction of the pathogen in the colonies. Arredondo et al. (2018) evaluated the potential beneficial effect of $A$. kunkeei on larvae and adult bees by decreasing the mortality associated with $P$. larvae infection in larvae, and the counts of Nosema ceranae spores from adult honey bees. In our experiment, we confirmed the inhibitory effect of A. kunkeei V18 on $P$. larvae tested in a previous in vitro experiment, as we obtained negative results in AFB fast tests and by cultivation on MYPGP agar 14 and 21 days after the first application of the probiotic supplement. In the control and the pollen groups the pathogen was detected by both methods throughout the experiment. In the tested apiary we found only one colony positive for M. plutonius, but without the clinical signs of EFB. After the application of probiotic A. kunkeei V18 pollen suspension for 3 weeks, M. plutonius was not detectable in the intestines of adult bees. Only few studies have investigated the inhibitory effect of LAB on M. plutonius (Killer et al., 2014; Wu et al., 2014), but both were performed only under in vitro conditions.

In this study we tested the biofilm-forming activity of honey bee and pollen LAB isolates and their inhibitory effect on $P$. larvae, as the most important prerequisites for their further probiotic use. We found out that biofilm formation is species and strain specific and can be modulated by the addition of various saccharides. Almost every isolate used in our study was able to form biofilm in the presence of the correct sugar. Apilactobacillus kunkeei V18 showed the strongest biofilm-forming activity potential after the addition of fructose. In addition, it was one of the three isolates that most strongly inhibited the honey bee pathogen P. larvae. Based on these results, we performed a pilot in vivo test in situ, on honey bee colonies positive for the presence of the causative agent of American foulbrood, where this isolate reduced the presence of P. larvae 2 weeks, and in one positive hive also the presence of $M$. plutonius 3 weeks after the first application of the pollen suspension containing A. kunkeei V18. However, these preliminary results should be verified in further experiments.

\section{ACKNOWLEDGEMENT}

This work was supported by VEGA 1/0505/19. 


\section{REFERENCES}

Alberoni, D., Gaggia, F., Baffoni, L. and Giola, D. D. (2016): Beneficial microorganisms for honey bees: problems and progresses. Appl. Microbiol. Biotechnol. 100, 9469-9482.

Al-Ghamdi, A., Ali Khan, K., Javed Ansari, M., Almasaudi, S. B. and Al-Kahtani, S. (2018): Effect of gut bacterial isolates from Apis mellifera jemenitica on Paenibacillus larvae infected bee larvae. Saudi J. Biol. Sci. 25, 383-387.

Alippi, A. M. and Reynaldi, F. J. (2006): Inhibition of the growth of Paenibacillus larvae, the causal agent of American foulbrood of honeybees, by selected strains of aerobic spore forming bacteria isolated from apiarian sources. J. Invertebr. Pathol. 91, 141-146.

Arredondo, D., Castelli, L., Porrini, M. P., Garrido, P. M., Eguaras, M. J., Zunino, P. and Antúnez, K. (2018): Lactobacillus kunkeei strains decreased the infection by honey bee pathogens Paenibacillus larvae and Nosema ceranae. Benef. Microbes 9, 279-290.

Badel, S., Bernardi, T. and Michaud, P. (2011): New perspectives for Lactobacilli exopolysaccharides. Biotechnol. Adv. 29, 54-66.

Barker, R. J. (1977): Some carbohydrates found in pollen and pollen substitutes are toxic to honey bees. J. Nutr. 107, 1859-1862.

Costerton, J. W., Stewart, P. S. and Greenberg, E. P. (1999): Bacterial biofilms: a common cause of persistent infection. Science 284, 1318-1322.

Daisley, B. A., Pitek, A. P., Chmiel, J. A., Al, K. F., Chernyshova, A. M., Faragalla, K. M., Burton, J. P., Thompson, J. G. and Reid, G. (2020): Novel probiotic approach to counter Paenibacillus larvae infection in honey bees. ISME J. 14, 476-491.

European Commission (2003): Regulation 1831/2003/EC on additives for use in animal nutrition, replacing Directive 70/524/ EEC on additives in feeding-stuffs. https://eur-lex.europa.eu/ legal-content/EN/TXT/?uri=CELEX\%3A32003R1831.

FAO/WHO (2002): Joint FAO/WHO Working Group Report on Drafting Guidelines for the Evaluation of Probiotics in Food. FAO/WHO, London, UK.

Ferreira, L., Sánchez-Juanes, F., García-Fraile, P., Rivas, R., Mateos, P. F., Martínez-Molina, E., González-Buitrago, J. M. and Velázquez, E. (2011): MALDI-TOF mass spectrometry is a fast and reliable platform for identification and ecological studies of species from family Rhizobiaceae. PLoS One 6, e20223.

Forsgren, E., Olofsson, T. C., Vasquez, A. and Fries, I. (2010): Novel lactic acid bacteria inhibiting Paenibacillus larvae in honey bee larvae. Apidologia 41, 99-108.

Giovannacci, I., Ermel, G., Salvat, G., Vendeuvre, J. L. and BellonFontaine, M. N. (2000): Physicochemical surface properties of five Listeria monocytogenes strains from a pork-processing environment in relation to serotypes, genotypes and growth temperature. J. Appl. Microbiol. 88, 992-1000.

Kaushik, J., Kumar, A., Duary, R., Mohanty, A. and Grover, S. (2009): Functional and probiotic attributes of an indigenous isolate of Lactobacillus plantarum. PLoS ONE 4, e8099.

Khangholi, M. and Jamalli, A. (2016): The effects of sugars on the biofilm formation of Escherichia coli 185p on stainless steel and polyethylene terephthalate surfaces in a laboratory model. Jundishapur J. Microbiol. 9, e40137.
Killer, J., Dubná, S., Sedláček, I. and Švec, P. (2014): Lactobacillus apis sp. nov., from stomach of honeybees (Apis mellifera), having an in vitro inhibitory effect on the causative agents of American and European foulbrood. Int. J. Syst. Evol. Microbiol. 64, 152-157.

Kubota, H., Senda, S., Nomura, N., Tokuda, H. and Uchiyama, H. (2008): Biofilm formation by lactic acid bacteria and resistance to environmental stress. J. Biosci. Bioeng. 106, 381-386.

Kuzyšinová, K., Mudroňová, D., Toporčák, J. and Molnár, L. (2012): In vivo testing of the efficacy of bee probiotic lactobacilli. Folia Vet. 56, 14-16.

Leathers, T. D. and Bischoff, K. M. (2011): Biofilm formation by strains of Leuconostoc citreum and L. mesenteroides. Biotechnol. Lett. 33, 517-523.

Lee, Y. K. and Salminen, S. (2008): Handbook of Probiotics and Prebiotics. 2nd ed. John Wiley \& Sons, Hoboken, USA. p. 5.

Maeno, S., Tanizawa, Y., Kanesaki, Y., Kubota, E., Kumar, H., Dicks, L., Salminen, S., Nakagawa, J., Arita, M. and Endo, A. (2016): Genomic characterization of a fructophilic bee symbiont Lactobacillus kunkeei reveals its niche-specific adaptation. Syst. Appl. Microbiol. 39, 516-526.

Maruščáková, I. C., Schusterová, P., Bielik, B., Toporčák, J., Bíliková, K. and Mudroňová, D. (2020): Effect of application of probiotic pollen suspension on immune response and gut microbiota of honey bees (Apis mellifera). Probiotics Antimicro. Prot. 12, 929-936.

Mathialagan, M., Johnson Thangaraj Edward, Y. S., David, P. M. M., Senthilkumar, M, Srinivasan, M. R. and Mohankumar, S. (2018): Isolation, characterization and identification of probiotic lactic acid bacteria (LAB) from honey bees. Int. J. Curr. Microbiol. App. Sci. 7, 894-906.

Mudroňová, D., Toporčák, J., Nemcová, R., Gancarčíková, S., Hajdučková, V. and Rumanovská, K. (2011): Lactobacillus sp. as a potential probiotic for the prevention of Paenibacillus larvae infection in honey bees. J. Apicult. Res. 50, 323-324.

Nath, A., Haktanirlar, G., Varga, Á., Molnár, M. A., Albert, K., Galambos, I., Koris, A. and Vatai, Gy. (2018): Biological activities of lactose-derived prebiotics and symbiotic with probiotics on gastrointestinal system. Medicina (Kaunas) 54, 18.

OIE (World Organisation for Animal Health) (2013): Manual of Diagnostic Tests and Vaccines for Terrestrial Animals. World Organisation for Animal Health, Paris, France.

Olson, J. K., Navarro, J. B., Allen, J. M., McCulloh, C. J., MashburnWarren, L., Wang, Y., Varaljay, V. A., Bailey, M. T., Goodman, S. D. and Besner, G. E. (2018): An enhanced Lactobacillus reuteri biofilm formulation that increases protection against experimental necrotizing enterocolitis. Am. J. Physiol. Gastrointest. Liver Physiol. 3, 408-419.

O’Toole, G. A., Pratt, L. A., Watnick, P. I., Newman, D. K., Weaver, V. B. and Kolter, R. (1999): Genetic approaches to study of biofilms. Methods Enzymol. 310, 91-109.

Patruica, S. and Mot, D. (2012): The effect of using prebiotic and probiotic products on intestinal microflora of the honeybee (Apis mellifera carpatica). Bull. Entomol. Res. 102, 619-623.

Peng, Y. S. (1981): Tolerance of lactose by free-flying adult worker honeybees. J. Apicult. Res. 20, 89-93.

Phillips, E. F. (1927): The utilization of carbohydrates by honeybees. J. Agric. Res. 35, 385-424. 
Ramasamy, K., Abdullah, N., Wong, M. C., Karuthan, C. and Ho, Y. W. (2010): Bile salt deconjugation and cholesterol removal from media by Lactobacillus strains used as probiotics in chickens. J. Sci. Food Agric. 90, 65-69.

Simoes, M., Bennett, R. and Rosa, E. A. S. (2009): Understanding antimicrobial activities of phytochemicals against multidrug resistant bacteria and biofilms. Nat. Prod. Rep. 26, 746-757.

Sutherland, I. W. (1972): Bacterial exopolysaccharides. Adv. Microb. Physiol. 8, 143-213.

Terraf, M. C., Juárez-Tomás, M. S., Nader-Marciás, M. E. and Silva, C. (2012): Screening of biofilm formation by beneficial vaginal lactobacilli and influence of culture media components. J. Appl. Microbiol. 113, 1517-1529.

Wu, M., Sugimura, Y., Iwata, K., Takaya, N., Takamatsu, D., Kobayashi, M., Taylor, D. M., Kimura, K. and Yoshiyama, M. (2014): Inhibitory effect of gut bacteria from the Japanese honey bee, Apis cerana japonica, against Melissococcus plutonius, the causal agent of European foulbrood disease. J. Insect Sci. 14, 129.

Yang, Y., Sreenivasan, P. K., Subramanyam, R. and Cummins, D. (2006): Multiparameter assessments to determine the effects of sugars and antimicrobials on a polymicrobial oral biofilm. Appl. Environ. Microbiol. 72, 6734-6742. 